\title{
Evaluation of Measurement Uncertainty for Absolute Flatness Measurement by Using Fizeau Interferometer with Phase-Shifting Capability
}

\author{
G. Moona*, R. Sharma, U. Kiran and K. P. Chaudhary \\ CSIR-National Physical Laboratory, Delhi, India
}

Received: 11 February 2014 / Accepted: 22 May 2014 / Published online: 9 August 2014

(C) Metrology Society of India 2014

\begin{abstract}
It is important to precisely measure flatness of the optical flats, as many industries use these as reference standards to ensure the quality of precision measurements and fabricated components. This paper describes identification of sources of error and measurement uncertainty evaluation for three flat test. Three flat test is used for absolute flatness measurement of optical flats, with the help of Fizeau interferometer (VerifireXP/D, with phase shift interferometry) established recently at National Physical Laboratory, India (NPL-I). The absolute profile of reference flat with higher accuracy can be determined using liquid level reference but liquid flat reference is more difficult to realize practically. Therefore three flat test is frequently adopted in standard interferometric measurements and traceability of this test can also be established by using a traceable laser head. This paper describes three flat method in detail along with observations and evaluation of measurement uncertainty as per ISO GUM is also done. Factors contributing to uncertainty of measurement of surface flatness have been indentified and detailed evaluation of uncertainty in measurements has been reported here.
\end{abstract}

Keywords: Absolute flatness; Phase shift interferometer; Three flat test; Measurement uncertainty; Traceability

\section{Introduction}

Flatness measurement of optical surfaces is one of the most significant tasks of optical measurements [1]. A flat surface can be described as the "plane wave front", which is one of the basic components in the treatment of wave-optics [2]. Flatness error is characterized by interferometric method using a high-quality reference surface of known flatness. Considering that the reference surface is better than the test surface and thus all the differences are counted for the test surface. Therefore, the accuracy of measurement is directly related to the quality of the reference flat. The $\mathrm{p}-\mathrm{v}$ flatness of a surface is maximum minus minimum perpendicular distance of the surface from the best fit plane. This interferometric method of flatness error measurement is a form of length measurement in which distances variation between a test surface and a reference surface is measured at many locations simultaneously. The phase differences between the two wavefronts (reflected and transmitted) result in fringe pattern and that is a direct indication of the form of the

*Corresponding author, E-mail: moonag@mail.nplindia.org component being tested with respect to the reference optic [3]. Absolute flatness measurement is an interferometric method to obtain the flatness of an optical surface independent of the flatness of reference flat used in the test. In this method, three flats in a series of configurations are measured. Then the flats are rotated and replaced during measurements in a prescribed order to overcome the error source from reference flat. The absolute testing is actually beneficial, when we use a tested flat as a reference surface during measurements. The data obtained is extremely accurate and it allows high-accuracy characterization of other optical surfaces. In usual flatness test the test surface is compared with the reference surface and flatness measurement can be done with reasonable accuracy, but if errors of test surface are similar to those of reference surface, then it is impossible to identify which flat contributes the error. Three flat test eliminates the reference flat caused errors [4]. Thus 3-flat method is ideal for calibrating master flats because it gives an "absolute" calibration. No master values are, needed and the process is relatively free of systematic errors. The measurement scheme is such that the bending profiles are removed leaving only the free form profiles. The more commonly used masters are recalibrated by this method 
occasionally to check on their stability [5]. In present work, we describe uncertainty in measurement for three flat test, done by using a Fizeau interferometer with phase sensitive detection capability. For Fizeau interferometer the measurement uncertainty depends on two major kinds of sources. One is uncertainty due to inteferometric measurements, which involves phase shift errors, environmental conditions and camera non linearity [6]. The other source is the uncertainty due to reference flat. To minimize the uncertainty due to reference flat, it is very important to know the absolute profile of reference flat and thus three flat test is conducted [7].

\section{Fizeaue Interferometer with Phase Sensitive Detection Capability}

A Fizeau interferometer with phase-shifting capability has been established at NPL India. Schematic diagram of Fizeau interferometer is shown in Fig. $1[8,9]$.

This flatness measuring interferometer works on the principle of Fizeau interferometer and the technique used is Phase shift interferometry [8]. In phase shift interferometry, piezoelectric transducers are used to move the transmission element forward and backward, causing constant phase variations between the reference wave front $W_{\mathrm{R}}(-x, y)$ and the measurement wave front $W_{\mathrm{T}}(x, y)$. The phase estimation process in PSI require a sequence of interference images $(\mathrm{N}) \geq 3$, each having a different phase offset $\alpha$. Each interference image is called a frame. The intensity of interference image in the two- beam approximation is given by

$I=A+B+2(A B)^{1 / 2} \cos (\theta+\alpha)$

where $I$ is the fringe visibility, $A$ and $B$ are the intensities of two beams, $\theta$ is the original phase difference between the two beams in the interferometer, and $\alpha$ is the additional phase step introduced by PZT.

Fig. 1 Fizeau interferometer using a laser source
The objective of surface profiling PSI is to measure the reflecting wave-front phase $\theta$. PSI algorithm uses even measurements of the intensities $I_{1}, I_{2}, I_{3}, I_{4}, I_{5}, I_{6}, I_{7}$ corresponding to additional phase steps of $-3 \alpha,-2 \alpha,-\alpha, 0$, $3 \alpha, 2 \alpha, \alpha$ is used [9]. We can write the intensity equations at each pixel as

$$
\begin{aligned}
& I_{1}=A+B+2(A B)^{1 / 2} \cos (\theta-3 \alpha) \\
& I_{2}=A+B+2(A B)^{1 / 2} \cos (\theta-2 \alpha) \\
& I_{3}=A+B+2(A B)^{1 / 2} \cos (\theta-\alpha) \\
& I_{4}=A+B+2(A B)^{1 / 2} \cos (\theta) \\
& I_{5}=A+B+2(A B)^{1 / 2} \cos (\theta+\alpha) \\
& I_{6}=A+B+2(A B)^{1 / 2} \cos (\theta+2 \alpha) \\
& I_{7}=A+B+2(A B)^{1 / 2} \cos (\theta+3 \alpha)
\end{aligned}
$$

These equations yields the result for calculating phase $\theta$ as

$\theta=\tan -\left\{\left[7\left(I_{3}-I_{5}\right)+\left(I_{1}-I_{7}\right)\right] /\left[8 I_{4}-4\left(I_{2}+I_{6}\right)\right]\right\}$

This method is being employed to drive flatness with traceability to wavelength. Laser, whose wavelength is traceable to SI unit Metre, serves as the length scale for the distance measurements.

In a Fizeau interferometer, two flats are kept facing each other and forming a cavity. The Interference fringe pattern measures the optical path difference (OPD) of the cavity, and thus the flatness. If the peak deviation is $a$ and the fringe spacing is $b$, then estimate $a / b$ visually (Fig. 2). The flatness error is $(a / b) \times(\lambda / 2)$.

The light source is a low-power, helium-neon laser. The laser beam is expanded to a four inch (or six inch) diameter and exits the interferometer through the aperture (Fig. 3). A transmission element or reference surface $S_{\mathrm{R}}(x, y)$, mounted in front of the aperture, reflects some of the laser light back into the interferometer, thus creating a reference

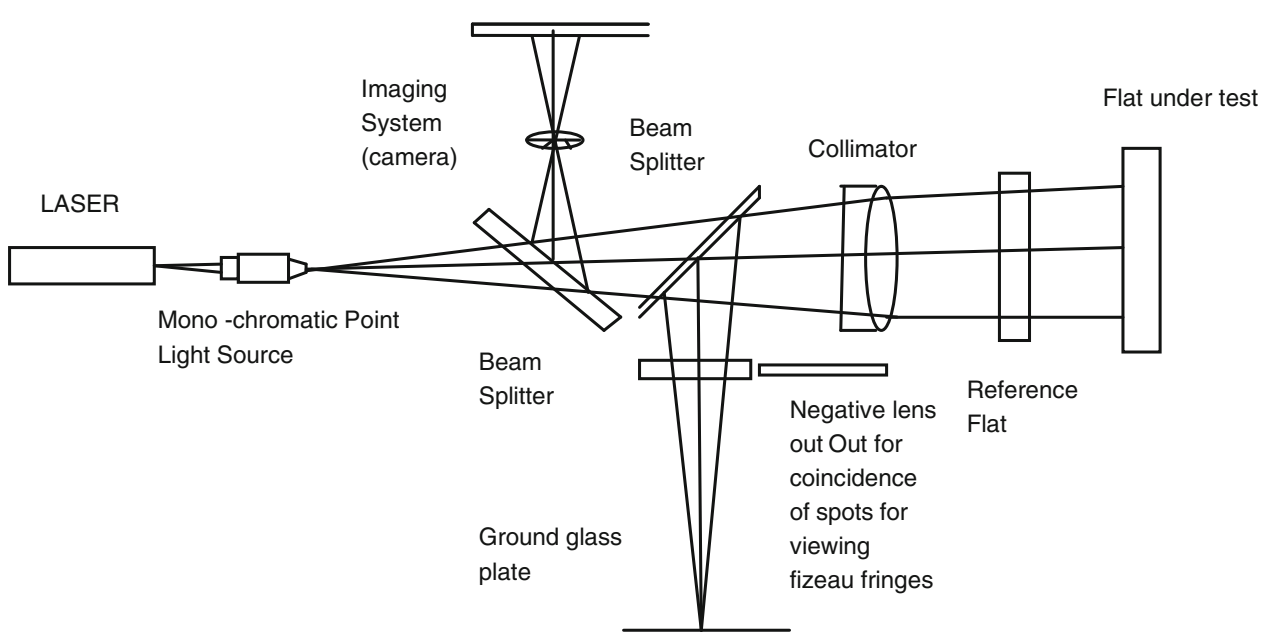




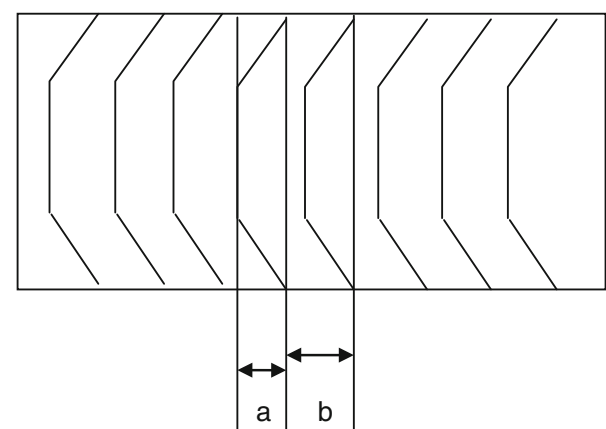

Fig. 2 Flatness measurement using conventional Fizeau interferometer

wavefront $W_{\mathrm{R}}(-x, y)$. The remainder of the laser light passes through the transmission element to the test surface $S_{\mathrm{T}}(x, y)$ and is referred to as the measurement wavefront $W_{\mathrm{T}}(x, y)$. When performing surface quality tests (Flatness error), the measurement wavefront $W_{\mathrm{T}}(x, y)$ reflects back to the interferometer from the test surface and recombined with the reference wavefront $W_{\mathrm{R}}(-x, y)$ and the two wavefronts interfere with each other. The phase differences between the two wavefronts result in an image of light and dark fringes that is a direct indication of the flatness error of the test and reference surface [10-12]. The interference pattern is converted to electrical signals by a video camera enabling software acquisition and analysis. In the coordinate system of the interferometer, the combined wavefront $W(x, y)$ is

$W(x, y)=W_{\mathrm{R}}(-x, y)_{+} W_{\mathrm{T}}(x, y)$

In Fizaeu interferometer, the flatness of the surface $S(x, y)$ is half of the wavefront error $W(x, y)$ :

$S(x, y)=\frac{1}{2} W(x, y)$

During a data acquisition sequence, the computer takes several "snapshots" of the interference pattern using CCD (Fig. 4), while introducing constant phase shift between the reference wave front $W_{\mathrm{R}}(-x, y)$ and the measurement wave front $W_{\mathrm{T}}(x, y)$. These snapshots are processed by the computer to determine the phase of the wavefront at each point.

Fig. 3 Setup for flatness measurement Zygo VerifireXP/D

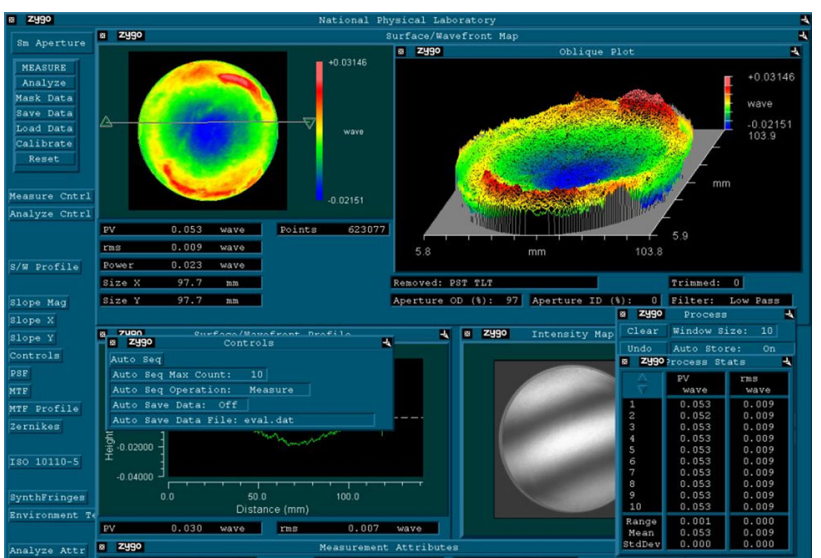

Fig. 4 Flatness measurements done by Zygo VerifireXP/D

The experimental procedure of three flat test consists of the measurement of one surface for each of the 3 flats. The surfaces to be measured in each sample are labeled as A, B and $\mathrm{C}$ (Fig. 5). Each surface is measured against the other two in prescribed measurement sequence and one of the samples is rotated 180 degrees and the measurements are taken [13]. The first three measurements provide absolute results along the vertical diameter. The fourth measurement with rotation facilitates to provide results along the horizontal diameter. The results of the three measurements which have the same form as Eq. 1 can be written in a matrix equation as [14]

$\left(\begin{array}{l}W_{\mathrm{A}+\mathrm{B}}(x, y) \\ W_{\mathrm{A}+\mathrm{C}}(x, y) \\ W_{\mathrm{B}+\mathrm{C}}(x, y)\end{array}\right)=\left(\begin{array}{llll}1 & 0 & 1 & 0 \\ 1 & 0 & 0 & 1 \\ 0 & 1 & 0 & 1\end{array}\right)\left(\begin{array}{c}W_{\mathrm{A}}(x, y) \\ W_{\mathrm{B}}(x, y) \\ W_{\mathrm{B}}(-x, y) \\ W_{\mathrm{C}}(-x, y)\end{array}\right)$

$W_{\mathrm{A}}, W_{\mathrm{B}}, W_{\mathrm{C}}$ are the wave fronts reflected by the three flats and $W_{\mathrm{A}+\mathrm{B}}, W_{\mathrm{A}+\mathrm{C}}, W_{\mathrm{B}+\mathrm{C}}$ are the measurements of the combined wave front errors.

Equation 4 can be split into invariant component $I(x, y)$ and the variant component $V(x, y) . I(x, y)=(I(-x, y)$ under reflection and $V(x, y)$ contributes to the uncertainty in measurements [15].

$W(x, y)=I(x, y)+V(x, y)$

So the matrix equations for absolute surface data of three flats A, B and C is reduced to [16].
Interferometer

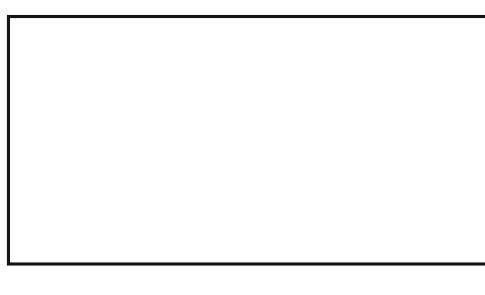

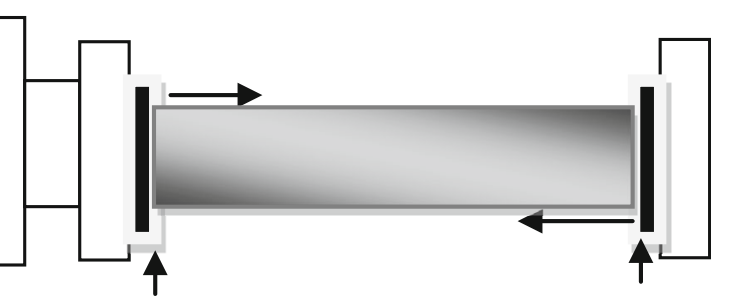

Reference

flat
Flat under test 


$$
\left(\begin{array}{l}
W_{\mathrm{A}}(x, y) \\
W_{\mathrm{B}}(x, y) \\
W_{\mathrm{C}}(x, y)
\end{array}\right)=\frac{1}{2}\left(\begin{array}{ccc}
1 & 1 & -1 \\
1 & -1 & 1 \\
-1 & 1 & 1
\end{array}\right)\left(\begin{array}{l}
W_{\mathrm{A}+\mathrm{B}}(x, y) \\
W_{\mathrm{A}+\mathrm{C}}(x, y) \\
W_{\mathrm{B}+\mathrm{C}}(x, y)
\end{array}\right)
$$

Equation resulting due to variant component which contributes the uncertainty in measurement and invariant component is as follows measurements provide coincident data also over the horizontal diameter [13].

While conducting experiment, it is to make sure that second surface of each sample should not be very parallel to the first surface to avoid the interference pattern between these surfaces. Alignment of the surfaces is done by adjusting the mechanical mounts on which the flats are mounted. Electronic filters are used to remove

$$
\left(\begin{array}{l}
W_{\mathrm{A}}(x, y) \\
W_{\mathrm{B}}(x, y) \\
W_{\mathrm{C}}(x, y)
\end{array}\right)=\frac{1}{2}\left(\begin{array}{cccccccc}
1 & 1 & -1 & 2 & -2 & 0 & 0 & 0 \\
1 & -1 & 1 & 0 & 0 & 0 & 2 & 0 \\
-1 & 1 & 1 & 0 & 0 & -2 & 2 & 2
\end{array}\right)\left(\begin{array}{c}
W_{\mathrm{A}+\mathrm{B}}(x, y) \\
W_{\mathrm{A}+\mathrm{C}}(x, y) \\
W_{\mathrm{B}+\mathrm{C}}(x, y) \\
\delta_{\mathrm{A}+\mathrm{B}}(x, y) \\
\delta_{\mathrm{BR}+\mathrm{c}}(x, y) \\
\delta_{\mathrm{A}+\mathrm{B}}(-x, y) \\
\delta_{\mathrm{BR}+\mathrm{c}}(-x, y) \\
\delta_{\mathrm{A}+\mathrm{c}}(-x, y)
\end{array}\right)
$$

\section{Experiment}

Three Flat Application is used to measure the absolute flatness error of a flat with respect to a reference flat, with a precision of, or better than, 1/100th of a wavelength. Three flat testing requires two transmission flats, a test flat, and a 2-axis mount (Fig. 5). Three series of measurements provide coincident data over a vertical diameter; four tilt and piston. After obtaining the fringe pattern the fringe nulling is done and measurements are taken (Fig. 6). The experimental procedure consists of the measurement of $\mathrm{A}+\mathrm{B}, \mathrm{A}+\mathrm{C}, \mathrm{B}+\mathrm{C}$ and $\left(\mathrm{B}^{\mathrm{R}}+\mathrm{C}\right)$ and the measurements are recorded $[16,17]$. To ensure that same area of surfaces is taken for measurements masking is done by using mask editor ( $\mathrm{X}$ pos: 491.5 pix and Y pos: 491.5 pix) (Fig. 7).

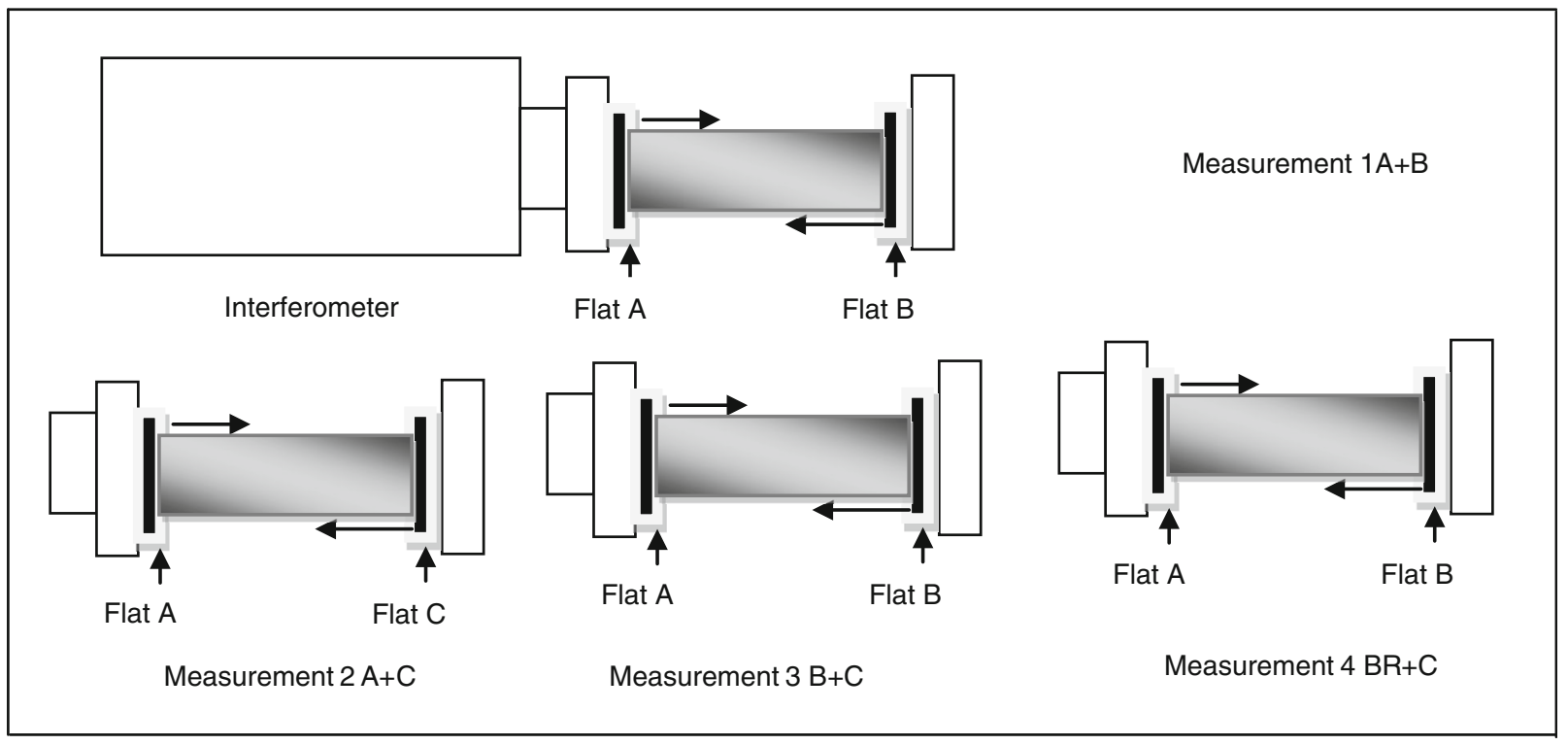

Fig. 5 Setup for Three flat test 


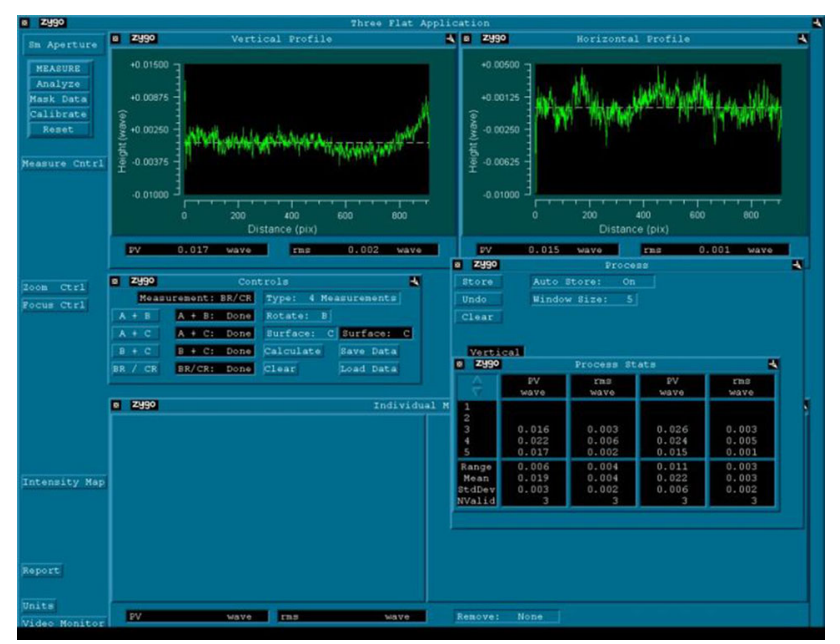

Fig. 6 Three flat test done by Zygo VerifireXP/D

\section{Result and discussions}

The environmental conditions during measurement were

Start: temperature $20.3{ }^{\circ} \mathrm{C}$ and relative humidity $52.6 \%$

End: temperature $20.27{ }^{\circ} \mathrm{C}$ and relative humidity $50.7 \%$
Three flats taken for measurements were zygo dynaflect with diameter $102 \mathrm{~mm}(\mathrm{~A})$, zygo transmission flat with diameter $102 \mathrm{~mm}$ ( B) and one test optical flat with diameter $152 \mathrm{~mm}(\mathrm{C})$. Five set of observations (Table 1) were taken to test the absolute flatness of test flat (C).

The software uses the following equations to find out the absolute surface flatness data along both the diameters (horizontal and vertical)

$$
\left.\begin{array}{rl}
\text { Flat } A & =[(A+B)+(A+C)-(B+C)] / 2 \\
\text { Flat } B & =[(A+B)+(B+C)-(A+C)] / 2 \\
\text { Flat } C & =[(A+C)+(B+C)-(A+B)] / 2
\end{array}\right\}
$$

Further uncertainty evaluation is done for three flat test. The error $E$ in flatness measurement $(W)$ can be evaluated from the model function given below [18-20]:

$$
\begin{aligned}
E= & W_{\mathrm{m}}-W_{\mathrm{a}}+\delta W_{\text {laser }}+\delta W_{\text {Air }}+\delta W_{\mathrm{T}}+\delta W_{\mathrm{M}}+\delta W_{\mathrm{F}} \\
& +\delta W_{\mathrm{PD}}
\end{aligned}
$$

$W_{\mathrm{m}}=$ Flatness measured, $W_{\mathrm{a}}=$ Actual flatness, $\delta W_{\text {laser }}=$ correction due to effect of environmental condition on laser wavelength, $\delta W_{\text {Air }}=$ correction due to air turbulence, $\delta W_{\mathrm{T}}=$ correction due temperature effects, $\delta W_{\mathrm{M}}=$ correction due to mechanical mounting,
Table 1 Result of measurements of three flat test

\begin{tabular}{llllll}
\hline S. No. & \multicolumn{2}{c}{$C, P V(\lambda)$} & & \multicolumn{2}{l}{$C, P V(\mathrm{~nm})$} \\
\cline { 6 - 6 } & Vertical profile & Horizontal profile & & Vertical profile & Horizontal profile \\
\hline 1 & 0.038 & 0.055 & 0.048 & 24.04 & 34.8 \\
2 & 0.034 & 0.049 & 21.52 & 30.37 \\
3 & 0.042 & 0.046 & 26.58 & 31.01 \\
4 & 0.040 & 0.051 & 25.31 & 29.11 \\
5 & 0.044 & & 27.84 & 32.27 \\
\hline
\end{tabular}

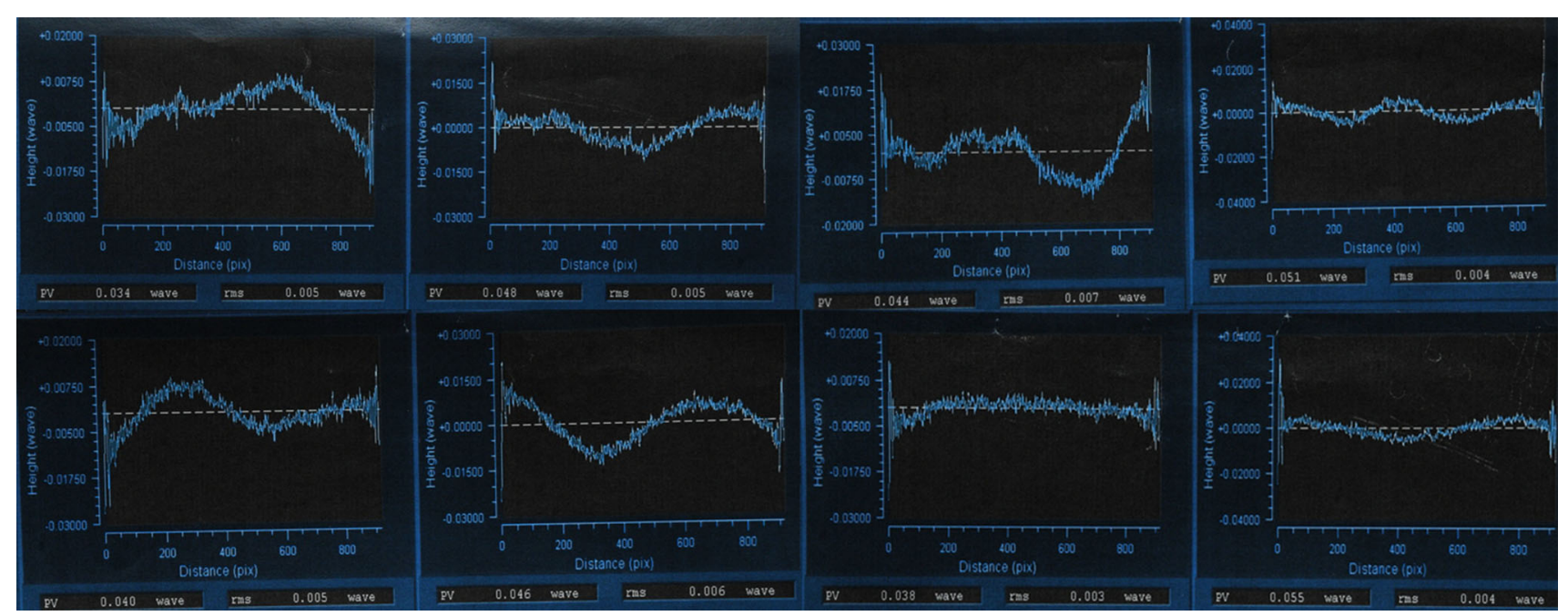

Fig. 7 Vertical and horizontal profiles of the test flat 


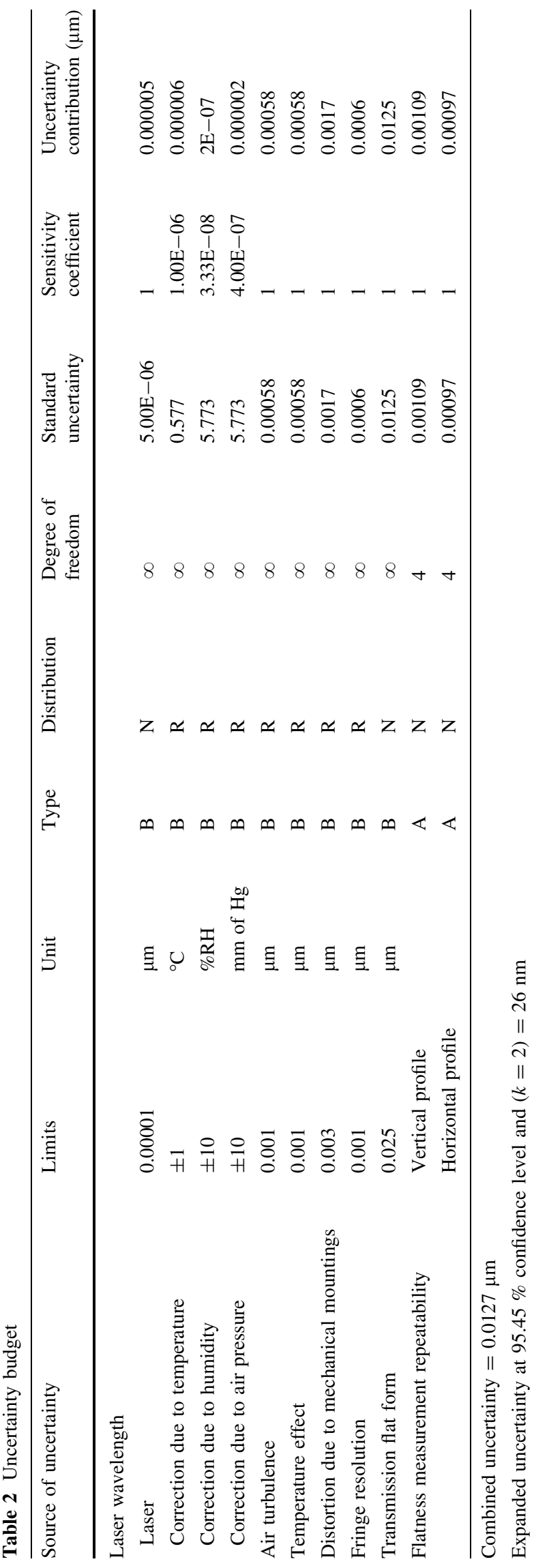

$\delta W_{\mathrm{F}}=$ correction due to transmission flat form(depends on flat used), $\delta W_{\mathrm{PD}}=$ correction due to fringe resolution.

Uncertainty budget for absolute flatness measurement is given in Table 2.

\section{Conclusions}

Absolute flatness testing is an interferometric method for testing the surface flatness of flats, independent of the reference flat used in test. We can utilize actual benefit of absolute testing when we use a tested flat as a reference surface during subsequent measurements and we have done the same by using a standard $102 \mathrm{~mm}$ reference flat of known flatness. This way we can get very accurate surface data resulting into high-accuracy characterization of other optical surfaces. Every measurement is desired to be repeatable, for lower measurement uncertainty. The evaluation of uncertainty is done keeping in view all evident factors. The expanded uncertainty comes out to be $26 \mathrm{~nm}$ at $k=2$. This work can be extended in future to reduce measurement uncertainty by working on above parameters and also identifying more parameters contributing towards measurement uncertainty.

Acknowledgments The authors would like to thank Prof. R. C. Budhani, Director, CSIR- National Physical Laboratory, New Delhi for his constant encouragement and support for this work. The Authors also acknowledge the financial support from CSIR.

\section{References}

[1] D. Malacara, M. Servin and Z. Malacara, Interferogram analysis for optical testing. Marcel Dekker, New York (1998). Copyright 2005 by Taylor and Francis.

[2] M. F. Küchel, A new approach to solve the three flat problem, Opt.-Int. J. Light Electron Optics, 112 (2001) 381-391.

[3] C. Xu, L. Chen and J. Yin, Method for absolute flatness measurement of optical surfaces. Appl. Optics, 48 (2009) 2536-2541.

[4] http://www.veeco.com/pdfs/appnotes/an506-absolute_05083_ 352.pdf.

[5] NBSIR 75-975- The calibration of an optical flat by interferometric comparison to a master optical flat.

[6] M. Bahrawi, N. Farid, Application of a commercially available displacement measuring interferometer to line scale measurement and uncertainty of measurement, MAPAN-J. Metrol. Soc. India, 25 (2010) 259-264.

[7] Y. Bitou, T. Takatsuji and K. Ehara, Simple uncertainty evaluation method for an interferometric flatness measurement machine using a calibrated test flat, Metrologia, 45 (2008) 21-26.

[8] http://photonics.intec.ugent.be/education/ivpv/res_handbook/ v2ch21.pdf.

[9] D. Bhattacharyya, A. Ray, B.K. Dutta and P.N. Ghosh, Direct measurement on transparent plates by using FIZEAU interferometry, Optics Laser Technol., 34 (2002) 93-96. 
[10] GPI \& Verifire operating manual OMP-0545F, Copyright 2009 by Zygo Corporation, USA.

[11] P. Hariharan, B.F. Oreb and T. Eiju, Digital phase shift interferometry: simple error-compensating phase calculation algorithm, Appl Optics, 26 (1987) 2504-2506.

[12] A.J. Damião, F.D. Origo, M.W. Ribeiro, and M.A. Fogarin Destro, Optical flats and optical parallels calibrated by interferometry, METROSUL IV (IV Metrology Latin American congress), Fozdo, Iguagu, Brazil (2005).

[13] GPI three flat application manual 0387_B note, Zygo Corporation, USA.

[14] U. Griesmann, Q. Wang and J. Soons, Three flat tests including mounting-induced deformations, Opt. Eng., 46 (2007) 093601.

[15] V. Greco, R. Tronconi, C. Del Vecchio, M. Trivi and G. Molesini, Absolute measurement of planarity with Fritz's method: uncertainty evaluation, Appl. Optics, 38 (1999) 2018-2027.
[16] A.J. Damião, F.D. Origo, M.A.F. Destro, M.W Ribeiro and R.A. Stempniak, Optical surfaces flatness measurements using the three flat method, XXVI ENFMC-Ann. Optics, 5 (2003).

[17] Three flat test application note, Zygo's reference manual.

[18] K.P. Chaudhary, C. Shakher and K. Shashi, Measurement of the size and spacing of standard wire sieves using an image processing system and wavelet transform, MAPAN-J. Metrol. Soc. India, 26 (2011) 15-27.

[19] K.P. Chaudhary, R. Sharma, F. Niveen and R.P. Singhal, Calibration of step gauge by substitution method on coordinate measuring machine and evaluation of uncertainty of measurement, MAPAN-J. Metrol. Soc. India, 17 (2002) 63-74.

[20] R. Sharma, K.P. Chaudhary, A.K. Kanjilal and V.G. Kulkarni, Estimation of uncertainty in measurement: application of ISO guidelines in case of measurements and calibration using laser interferometers, MAPAN-J. Metrol. Soc. India, Suppl. Issue 1 (2001) 